\title{
Putting and Taking Events in Mandarin Chinese
}

\author{
JIDONG CHEN \\ California State University at Fresno
}

\section{$1 \quad$ Introduction}

Placement events involve the caused change of location of an object, putting it at or removing it from a location (i.e. a "putting" or a "taking" event). These events, typically described in English by a simplex verb such as take, as in She took the box from the bookshelf, are typically encoded in Mandarin with a verb compound such as na2-xia4-lai2 'take-descend-come', as in (1):

\section{(1) Ta1 cong2 shuljia4 shang4 na2-xia4-lai2 she from bookshelf on ${ }^{1}$ take-descend-come na4 ge4 he2zi. ${ }^{2}$ \\ that CLF box}

'She from the bookshelf took down the box (towards herself).'

The first verb $\left(\mathrm{V}_{1}\right)$ of the verb compound, na2 'take', encodes the general action of removing, the second verb $\left(\mathrm{V}_{2}\right)$, xia4 'descend', encodes the Path of motion, and the third verb $\left(\mathrm{V}_{3}\right)$, lai2 'come', indicates Deictic information (i.e. towards the Agent in [1]). Verb compounds like na2-xia4-lai2 are known as directional verb compounds, which typically describe motion events and involve a verb indicating the Path of motion.

This paper investigates the lexical semantics of Mandarin placement predicates from the perspective of event encoding and event categorization. The data explored in this study are the verbs used in elicited descriptions of 63 Put\&Take video clips (Bowerman, Gullberg, Majid, andNarasimhan, 2004) plus three warm-up items, depicting a wide range of caused putting and taking events by 10 adult native speakers of Mandarin (mean age 28 years). In $\S 2$, I give an overview of the grammatical encoding of the semantic components of a placement event in a clause - Figure, Ground, Motion, and Path. In §3, I discuss the lexical semantics of verb compounds and their component verbs. I

\footnotetext{
${ }^{1}$ The gloss for shang4 'on/top' is adopted for convenience of discussion as there is no exact counterpart in English.

${ }^{2}$ The numbers mark tones. Abbreviations used in the glosses in this paper include: PFV = perfective, $\mathrm{CLF}=$ classifier, GEN $=$ genitive/possessive case marker.
} 


\section{Jidong Chen}

first examine Path verbs since Path is the key component of a motion event. Mandarin Path verbs are often in the second or third position in a directional verb compound and they constitute a closed set. I then illustrate the event categories selected by verbs that have been used in the $\mathrm{V}_{1}$ slot of a directional verb compound and specify the crucial semantic features for these distinctive categories.

\section{Encoding Placement Events}

A placement event, as a particular type of motion event, is analyzed as having four basic semantic components, Motion, Ground, Figure, and Path (Talmy, 1985, 2000). ${ }^{3}$ In Mandarin the information about Motion and Path is encoded in the predicates (see $\$ 3$ for details). The Ground and Figure components are encoded as arguments or adjunct coverb phrases in the clause. ${ }^{4}$

The Ground information includes the location from which the Figure moves (i.e. Source) and at which the Figure ends up (i.e. Goal). The Source information always occurs in a preverbal position as an adjunct coverb phrase, e.g. cong2 shui3 li3 'from water inside' (= from the water), as in (2). The coverb cong2 is a preposition-like morpheme and it must be used together with a spatial particle such as li3 'inside' or shang4 'on' after the Ground NP. It is the spatial particle that explicitly indicates the spatial relation between the Figure entity and the Ground entity. ${ }^{5}$ It is ungrammatical to leave the spatial particle out, e.g. *zai4 di4 'at ground' (cf. zai4 di4 shang4 'at ground on' in [2]). To encode the spatial relations between the Figure and the Gound that is typically encoded with a preposition such as in and on in English, Mandarin utilizes two components, a coverb, as a general locative marker, and a locative particle.

(2) Ta1 ba3 zhuan1tou cong2 shui3 li3
she BA brick from water inside take-exit-come
'She took the brick from the water.'

\footnotetext{
${ }^{3}$ I follow Talmy's (Talmy, 2000) theoretical framework in analyzing motion events and adopt the following terms to refer to:

Figure $=$ the object that undergoes a change of location

Ground $=$ a reference with reference to which the path, site, or orientation of the Figure is

characterized

Source $=$ origin of the motion of the Figure

Goal $=$ orientation of the motion of the Figure

Agent $=$ the causer of motion

Path $=$ trajectory or Deixis of motion

${ }^{4} \mathrm{Li}$ and Thompson (1981) use "coverb" to refer to a group of morphemes that may function as a verb in some contexts but that can also behave like functional words in other contexts in Mandarin. Typical coverbs include morphemes like zai4 'at', cong2 'from', gen1 'with', cong2 'from', chao2 'facing', yan2 'along', and li2 'be apart from'.

${ }^{5}$ Morphemes like li3 'inside' or shang4 'on' were called "locative particles" (Li and Thompson, 1981). Other locative particles are xia4 'under', wai4 'outside', qian2 'front', hou4 'back', pang2 'side', and zhongljian1 'middle'.
} 
The Goal information always occurs at a post-verbal position either as an adjunct coverb phrase such as zai4 di4 shang4 'at ground on' in (3) or as an NP of the complex predicate such as tal de tou2fal li3 'her hair inside' (= the inside part of her hair) as in (4).

\section{(3)}

$\begin{array}{llllll}\text { Tal ba3 mu4kuai4 dao4 zai4 di4 } & \text { shang4. } \\ \text { he } & \text { BA wood.block pour at ground } & \text { on } \\ \text { 'He poured the wooden blocks on the ground.' } & \end{array}$

\section{(4)} she BA flower insert-enter PFV she GEN hair inside 'She inserted the flower into her hair.'

The Figure, i.e. the entity that undergoes the movement, is encoded as the argument of the predicate (as in [1]). It also occurs as an NP following the morpheme $b a 3$ (henceforth the BA NP), as in (2), (3), and (4). The morpheme ba3 was a full verb in classical Chinese, meaning 'take, hold, handle' (e.g., Bennett, 1981; Wang, 1954), but it has become grammaticalized and its original status as a full verb has weakened in modern Chinese. Constructions containing the morpheme ba3 are commonly referred to as "the BA construction", in which the referent of the BA NP usually receives a reading of being disposed of, dealt with, manipulated or handled, and, thus, affected by the action denoted by the VP in the clause. This meaning gives the BA construction the label "disposal construction" (Chao, 1968; Li and Thompson, 1981; Wang, 1954). ${ }^{6}$

In the elicited descriptions of placement events, $75 \%$ (472 out of the total of 630) of the constructions consists of BA constructions. This dominance may be due to three factors. First, the "disposal or manipulation" meaning of the BA construction corresponds to the manipulation meaning and the caused change of location of the entity in a placement event. Second, Mandarin is argued to favor temporal iconicity (Haiman, 1985; Tai, 1985) and the order of the grammatical components in the BA construction conforms indeed to this principle. That is, the surface order of the syntactic units matches the temporal order of the placement events - holding or handling the object and then placing it at or removing it from a location. This temporal sequence is realized syntactically with the morpheme ba3 followed by the Figure NP that is followed by the placement verb. The historical meaning of ba3 as 'take, hold, handle' is still detectable in the BA construction. The counterparts of ba3 in a number of south eastern Asian languages have preserved their full verb status and are frequently used with other placement verbs to form a serial verb construction to describe placement events (Enfield, p.c.). Finally, ba3 is syntactically required to allow more than one constituent to occur in a postverbal position. Mandarin restricts the number of constituents post-verbally

\footnotetext{
${ }^{6}$ The status of $b a 3$ is controversial: it has been argued to be a case marker (Huang, 1982), a focus marker (Sun and Givón, 1985), or a secondary topic marker (Tsao, 1996).
} 


\section{Jidong Chen}

and when more than one constituent is placed in the post-verbal position, the sentence becomes ungrammatical, e.g. *tal chal-jin4 hual tal de tou2fal li3 'she insert-enter flower her hair inside', where two phrases, hual 'flower' and tal de tou2fal li3 'her hair inside' occur after the verb compound chal-jin4 'insert-enter'. When ba3 is used, however, the NP encoding the Figure follows ba3 directly and thus leaves only the Goal phrase post-verbally, as in (3) and (4).

\section{$3 \quad$ Lexical Semantics of Placement Verbs}

The meanings of Motion and Path are encoded in compound placement predicates in Mandarin. In this data set, $67 \%$ of all the verb tokens are verb compounds and the remainder consists of simplex verbs. All the simplex placement verbs are also used as the $\mathrm{V}_{1}$ of a verb compound. ${ }^{7}$ It is worth noting that not all the elicited verbs of manipulation $\left(\mathrm{V}_{1}\right)$ by themselves encodes the core meaning of a placement, i.e. caused change of location, such as diaol 'hold in mouth', jia2 'hold tightly'. The caused motion meaning is conveyed by the compound as a whole, i.e. when such a verb of manipulation is combined with a $\mathrm{V}_{2}$ indicating Path of motion. This resembles the so-called English caused motion construction (e.g., Goldberg, 1995) such as John squeezed the ball into the box, where the verb squeeze by itself does not have a motion meaning. Therefore, in these cases the constructional meaning of verb compound, rather than the verb alone, gives rise to the caused motion reading. In what follows, I discuss the composition of verb compounds and the lexical semantics of the high-frequency simplex placement verbs.

\subsection{Productivity and the Semantic Composition of the Directional Verb Compounds}

Verb compounding is a very productive process in Mandarin, involving the combination of two or three free verbs in a fixed order, e.g. na2-xia2-lai2 'take-descend-come', as in (1). ${ }^{8}$ The first verb $\left(\mathrm{V}_{1}\right)$ usually encodes the manipulation of an object (e.g. putting or taking), the second verb $\left(\mathrm{V}_{2}\right)$ the Path of the motion, and the third verb $\left(\mathrm{V}_{3}\right)$ the Deictic relation between the Agent (or the speaker) and the direction of motion of the Figure object. ${ }^{9} \mathrm{~A}$ directional verb compound $\left(\mathrm{V}_{1} \mathrm{~V}_{2}\right.$ or $\left.\mathrm{V}_{1} \mathrm{~V}_{2} \mathrm{~V}_{3}\right)$ clearly represents a placement event as composed of two crucial semantic components, each encoded by one verb. Each component verb is a full verb by itself and can be used freely outside verb compounds. The ordering of the component verbs is fixed and

\footnotetext{
${ }^{7}$ Simplex placement verbs were used only for putting events in the data. When a simplex verb was used, it was always immediately followed by a coverb locative phrase with zai4 indicating the Goal, e.g. zai4 di4 shang4 'at ground on' (= on the ground), as in (3).

${ }^{8}$ Mandarin verb compounds encode not only motion events but also change-of-state.

${ }^{9}$ Two verbs encoding Path were found in $\mathrm{V}_{1}$ position in this data set: shen 1 'to extend, to stretch' and diao4 'to fall'. In the context of these verbs, the role of $\mathrm{V}_{2}$ is to provide details about the Path followed by the Figure. See $\$ 3.4$ for details.
} 
iconic - the verb expressing the manipulation of an object (such as placing or taking) usually precedes the verb expressing the Path of motion.

The productivity of verb compounding was attested by the diversity of directional verb compounds used in the descriptions of placement events: A total of 59 types of verb compounds were produced (token frequency 423). This productivity is also revealed by the diversity of the verbs $\left(\mathrm{V}_{1}\right)$ that can combine with the Path verbs. A total of 37 types of $V_{1}$ were used by the ten speakers. These verbs were combined flexibly with one or two Path verbs which form a closed set in Mandarin.

\subsection{Path Verbs: $V_{2}$ and $V_{3}$ of the Directional Verb Compound}

Path is the key component of motion. In Mandarin Path information is typically encoded with one or two verbs $\left(\mathrm{V}_{2}\right.$ or $\left.\mathrm{V}_{3}\right)$ in a directional verb compound. The high frequency of verb compounds indicates that Path is often spelled out explicitly in the description of placement events.

Path verbs include verbs that indicate the trajectory of the motion such as shang4 'ascend' (up), and xia4 'descend' (down), and deictic verbs that indicate the orientation of motion such as lai2 'come' and qu4 'go'. The trajectory Path verbs can combine with the deictic verbs to form compounds. Table 1 shows the two groups of verbs and their combinations. ${ }^{10}$

Table 1. Mandarin Path Verbs and Their Combinations

(Note: Notations such as $\mathrm{V}_{2}$ or $\mathrm{V}_{3}$ indicate the position of a Path verb in a directional verb compound with three component verbs.)

\begin{tabular}{|c|c|c|}
\hline $\mathrm{V}_{2}-\mathrm{V}_{3}$ & lai2 'come' & qu4 'go' \\
\hline shang4 'ascend' & $\begin{array}{l}\text { shang4-lai2 } \\
\text { 'ascend-come' (come up) }\end{array}$ & $\begin{array}{l}\text { shang4-qu4 } \\
\text { 'ascend-go' (go up) }\end{array}$ \\
\hline xia 'descend' & $\begin{array}{l}\text { xia4-lai2 } \\
\text { 'descend-come' (come down) }\end{array}$ & $\begin{array}{l}\text { xia4-qu4 } \\
\text { 'descend-go' (go down) }\end{array}$ \\
\hline jin4 'enter' & $\begin{array}{l}\text { jin4-lai2 } \\
\text { 'enter-come' (come in) }\end{array}$ & $\begin{array}{l}\text { jin4-qu4 } \\
\text { 'enter-go' (enter) }\end{array}$ \\
\hline chul 'exit' & $\begin{array}{l}\text { chu1-lai2 } \\
\text { 'exit-come' (come out) }\end{array}$ & $\begin{array}{l}\text { chul-qu4 } \\
\text { 'exit-go' (go out) }\end{array}$ \\
\hline hui2 'return' & $\begin{array}{l}\text { hui2-lai2 } \\
\text { 'return-come' (come back) }\end{array}$ & $\begin{array}{l}\text { hui2-qu4 } \\
\text { 'return-go' (return) }\end{array}$ \\
\hline guo4 'pass' & $\begin{array}{l}\text { guo4-lai2 } \\
\text { 'pass-come' (come over) }\end{array}$ & $\begin{array}{l}\text { guo4-qu4 } \\
\text { 'pass-go' (go over) }\end{array}$ \\
\hline zou3 'be.away' & - & 一 \\
\hline qi3 'rise' & $\begin{array}{l}\text { qi3-lai2 } \\
\text { 'rise- come' (come up) }\end{array}$ & - \\
\hline
\end{tabular}

${ }^{10}$ The verb zou3 by itself means 'walk', but when it is used as the $\mathrm{V}_{2}$ in a compound it has only a gramamticalized meaning of 'be away'. For example, na2-zou3 'take-walk' means 'take away'. 
Jidong Chen

Note that neither a trajectory Path verb nor a deictic verb by itself encodes the meaning of placement. They have to combine further with a verb of manipulation to describe the placement events.

The two deictic verbs, lai2 'come' and qu4 'go', are optional - only $37 \%$ (158/423) of the verb compounds contain a deictic verb. Among these verb compounds, lai2 'come' is dominant (97\%). Lai2 'come' indicates the direction of motion of the Figure object towards the Agent of the action or towards the speaker who describes the event. For example, lai2 is used in na2xia4-lai2 'take-descend-come', as in (1), since the box is removed from the bookshelf (a higher location) to a lower location where the Agent is standing and the motion is oriented toward the Agent.

\subsection{Verbs Expressing Manipulation: $V_{1}$ of the Directional Verb Compounds}

Two general placement verbs, fang4 'put' and na2 'take', are the most frequent $\mathrm{V}_{1}$ of directional verb compounds. They are general in the sense that they have relatively little meaning beyond a schematic meaning of "manipulate a Figure object to cause motion/change of location". Events that were described with fang4 'put' or na2 'take' in this data set form a subset of the putting and taking events that are typically described with put or take in English (Bowerman, Majid, Gullberg, and Narasimhan 2004). Many placement events that fall into the extensional categories of English put and take are obligatorily encoded in Mandarin with placement verbs that convey more specific information than simply the bare schema of putting or taking. I call these verbs "specific verbs". The general placement verbs fang4 or na2 are often not applicable or not the most favored to describe the events depicted by the specific verbs. When a specific verb is used there is often a high degree of agreement among the speakers. The subsections below illustrate the extensional patterns of the general placement verbs fang4 and na2 and that of the specific verbs. Table 2 summarizes the crucial semantic distinctions that Mandarin speakers make in describing placement events.

\subsubsection{Fang4 'put'}

The verb fang4 'put' describes a large group of placement events such as 'putting cup on table', 'putting box up on shelf', and 'putting book on floor'. 14 of the total of 36 putting events were described with fang 4 with a $100 \%$ agreement on the use of this verb for most of the clips. These events involve putting a large range of inanimate Figure entities with different physical properties, i.e. cup, rice, box, book, apple, stone, pen, and rope, at various kinds of locations such as table, shelf, floor, tree branch, and hole. Although the current set of stimuli does not include any animate Figure entities, fang 4 can also be used for such cases, e.g. putting a baby into cradle. The resultant orientation of the Figure can be either standing (such as cup on table) or lying 
(such as book on floor). The verb fang 4 was also used to describe placing an object with an instrument, e.g. putting a banana on table with long tongs. Given such wide range of uses, fang4 'put' is obviously a broad 'put-type' verb with no entailments regarding the specific physical properties of Figure or Ground, the resultant orientation of the Figure, and Manner of manipulation.

\subsubsection{Na2 'take'}

The verb na2 'take' describes a large range of events of removing a Figure from a Ground: $48 \%$ of all the taking events (13 of the total of 27) were described with $n a 2$, with an average of $85 \%$ agreement among the speakers. These events consist of removing different types of inanimate Figure entities such as box, orange, stone, rope, poster and stone from various kinds of locations such as table, self, box, tree branch, as well as from an animate Ground (e.g. taking a coke can from someone).

Interestingly, for 6 out of 27 taking events, some speakers described these events with specific verbs such us ba2 'pull' for taking cucumber out of a recorder case, to focus on a tight-fitting relation between the Figure and the Ground. In these specific contexts, $n a 2$ is clearly less frequently selected, if not considered inappropriate by Mandarin speakers.

\subsubsection{Verbs of Dressing and Undressing}

Specific dressing or undressing verbs typically describe putting clothes on or taking them off. Mandarin has three such verbs, chuan1 'put on', dai4 'put on', and tuol 'take off'. These verbs were used by all the speakers across the same set of events - events of putting on or removing clothes - with $100 \%$ agreement. Sample descriptions are shown in (5) and (6).

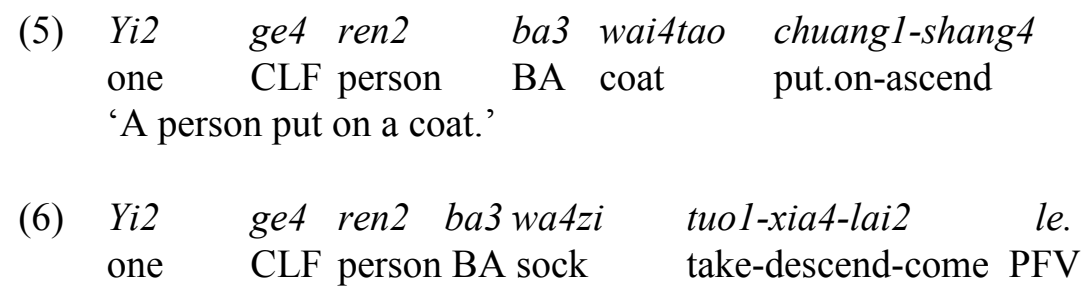

'A person took off her socks.'

The two putting-on-clothing verbs, chuanl and dai4, are selected according to the body part (i.e. the Goal) or the entities involved: chuanl is used only for putting on clothing on the trunk of the body, e.g. putting on a coat, putting on trousers; dai4 is used for putting on accessories such as hat, earrings, necklace, bracelet, glasses, or ring. These entities typically land on the joints or the extremities of the body such as wrist, finger, or ears. The verb tuol has a general meaning of removing clothing off of body parts and is applicable to the removing of any kind of clothing (including small accessories) from any part of the body. The general verb fang4 'put' cannot be used for putting 


\section{Jidong Chen}

clothing or accessories on body parts. In contrast, the general verb na4 'take' may be used for removing small accessories from body part, e.g. taking off hat (from head).

\subsubsection{Verbs Specifying Spatial Relations between Figure and Ground}

The relation between Figure and Ground plays a role in determining the extensional categories of the placement verbs. Two putting verbs, sail 'stuff' and chal 'insert', and two taking verbs, ba2 'pull' and choul 'pull, draw', describe putting a Figure in or taking it from tight-fitting containment. The verbs sail is used only for soft (usually fluffy) objects, and chal only for rigid objects, as in (7) and (8).
(7) Yi2 ge4 ren2 ba3 bu4 sail-jin4
one CLF person BA rag stuff-enter
qi4che1 pai2qi4-guan3.
car exhaust-pipe

'A person stuffed a rag into the exhaust pipe of the car.'

(8) Na4 ge4 ren2 ba3 na4zhu2 cha1-jin4 zhu2-tai2. that CLF person BA candle insert-enter candle-stand 'That person inserted the candle in the candle stand.'

The verbs ba2 'pull' and choul 'pull, draw' both describe removing a Figure from a tight-fitting container; choul, in particular, is used for pulling a long object from a tight-fitting container. This is illustrated in (9) and (10).

(9) Na4 ge4 ren2 ba3 na4zhu2 cong2

that CLF person BA candle from

zhu2tai2 shang4 ba2-xia4-lai2.

candle.stand on pull-exit-come

'That person inserted the candle in the candle stand.'

$\begin{array}{lllll}\text { (10) Ta1 cong2 } & \text { dai4zi } & \text { li3 } & \text { choul-chu4 huanglgual. } \\ \text { he } & \text { from } & \text { bag } & \text { inside } & \text { pull-exit cucumber }\end{array}$

'He pulled a cucumber from the bag.'

Mandarin distinguishes tight-fitting relation from adhesive relationship. The verb tiel 'stick' describes attaching a Figure, which is subject to gravity, to the Ground with adhesives such that it stays on the Ground, as shown in (11).

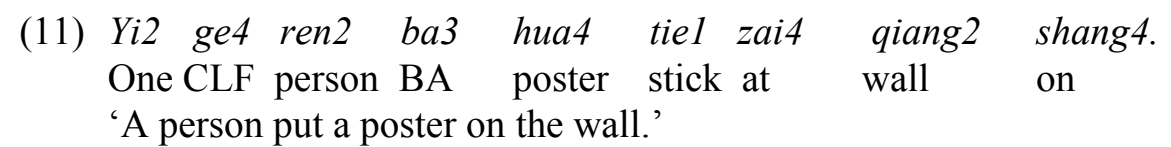


Mandarin also distinguishes removing a Figure from a lower location, as represented by the verb jian3 'pick' in (12).

(12) Ta1 cong2 di4 shang4 jian3-qi3 yil ben3 shu1. she from floor on pick-rise one CLF book 'She picked up a book from the floor.'

Nine of the ten speakers used jian3 'pick' to describe the picking event, and only one speaker used na2 'take'. When probed whether na2 was also appropriate to describe this event, all the speakers agreed that na2 was applicable. It seems that even if the general taking verb $n a 2$ is available and appropriate to describe such an event, it is not preferred; rather a specific verb is favored as it conveys more information than just manipulation.

\subsubsection{Verbs Specifying Manner}

Two taking verbs, taol 'draw, dig' and zhual 'grasp, hold tightly', specify the Manner in which the Agent removes a Figure from a Ground, as in (13) and (14).

(13) Na1 ge4 ren2 cong2 kou3dai4 li3
That CLF person from pocket in
taol-chu1 yi2 mei2 ying4bi4.
take-exit one CLF coin
'That person took a coin from her pocket.'

(14) Tal cong2 zhuol shang4 zhual-qi3 yil ba3 dou4zi. she from table on grasp-rise one CLF bean 'She took a handful of beans from the surface of the table.'

The verb taol describes taking out a Figure in a scooping manner, usually after searching in a Ground object that is deep, e.g. the pocket as in (13) which encloses both the Figure object (coin) and the Agent's hand. The verb zhual 'grasp, hold tightly' describes the Agent opening her hand, taking hold of the Figure, and grasping it in the hand.

\subsubsection{Verbs Specifying Intentionality and Control}

Mandarin distinguishes intentional (controlled) vs. accidental placement, as reflected in four specific verbs - diao4 'drop', rengl 'throw', sa3 'spill', and dao4 'pour'. The events picked out by these verbs cannot be described with a general verb fang4 'put', and the speakers show a high degree of agreement on the use of each verb for the relevant stimuli.

The verb diao4 describes unintentional placing or dropping of a Figure, e.g. 'dropping book accidentally on floor'. Deliberate dropping, in contrast, is 


\section{Jidong Chen}

described with rengl 'throw', e.g. tossing a book on floor. This contrast is shown in (15) and (16).

(15) Yil ben3 shu1 diao4 dao4 di4 shang4 le. one CLF book fall to ground on PFV 'A book fell on the ground.'

(16) Tal ba3 shul rengl zai4 di4 shang4.

she BA book throw at ground on

'She threw the book on the ground.'

The verb $s a 3$ is typically used for accidentally spilling liquids, as in (17). It is also appropriate to describe accidental dropping of solid objects consisting of fine grains or particles, like sand, rice, flour, sugar or seeds.

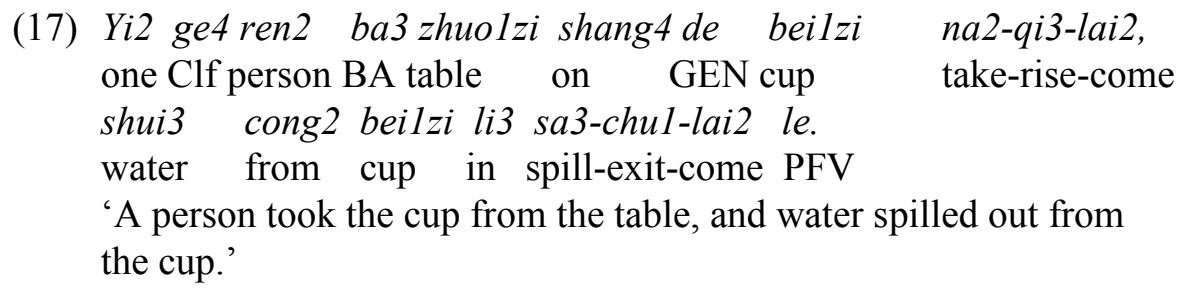

The verb dao4 'pour' typically describes the Agent either pouring liquid or dropping solid objects (e.g. wooden blocks) into a Ground object. Whereas in English such events are encoded with three English verbs, pour, dump, and flip, in Mandarin dao 4 covers all the events categorized by these three English verbs. This suggests that Mandarin does not distinguish placing liquid versus solid objects into a container; nor does it care about the Manner of pouring, e.g. flipping blocks off notepad into bowl.

\subsubsection{Verbs Encoding Instrument of Placement}

In the elicited data, six verbs encode instrument with which the placement action is conducted. These verbs are used for both putting and taking events: bao4 'hold in arm(s)', ling1 'carry by hand(s)', ti2 'carry by hand(s)', xian2 'hold in mouth', diaol 'hold in mouth', and jia2 'hold tightly (in instrument)'. The first five verbs typically entail a body part engaged in the placement action - $\operatorname{arm}(\mathrm{s})$ or arm-like instrument for bao4, hand(s) or hand-like instrument for ling1 and ti2 (which are synonyms), and mouth for xian 2 and diaol (which are synonyms). The verb jial entails holding a Figure tightly inbetween the instrument, e.g. holding a banana with long tongs.

\subsection{Verbs encoding Path as the $V_{1}$ of the Directional Verb Compounds}

Recall that Path is usually encoded with a second or third verb in a directional verb compound. There are, however, two verbs in this data set that entail Path, 
and that occur in the $\mathrm{V}_{1}$ slot of directional verb compounds, diao4 'fall' and shen1 'extend'. The verb diao4 'fall', describes accidental dropping of a Figure (cf. §3.3.6), and entails a downward motion as effected by gravity. This verb can combine with a Path verb that explicitly spells out downward motion entailed in diao4 'fall', e.g. diao4-xia4 'fall-descend', but it cannot combine with Path verb that entails a conflicting Path, e.g. *diao4-shang4 'fall-ascend'.

The verb shen1 'extend, stretch' can describe either putting or taking events. It entails a Figure's stretching out, which as a result ends up in a Ground object, e.g. 'putting head into and taking it out from a bucket' or 'taking one's hand from a hole on a tree', as in (18) and (19).

\section{(18) Ta1 ba3 tou2 shen1-jin4 le tong3 li3. \\ $\mathrm{He} \quad \mathrm{BA}$ head extend-enter PFV bucket in}

'He extended his head into the bucket.'

(19) Ta1 ba3 shou3 cong2 shu4dong4 li3 shen1-chul-lai2 le. she BA hand from tree.hole in extend-exit-come PFV 'She took her hand from the hole on the tree.'

The verb shen 1 is used only for events where a Figure extends itself and ends up in the space of a container. In other words, the part that moves is an integral part of the Agent that instigates the movement. When being probed whether fang4 'put' could be used to describe events as depicted by (18) or (19), most of the speakers rejected it. It appears therefore that the use of shen1, instead of fang 4 or $n a 2$, is specific to putting or removing a part of the Agent body.

\subsection{Summary}

I have shown the lexical semantics of the verbs that are typically the $\mathrm{V}_{1} \mathrm{~s}$ of a directional verb compound in the elicited descriptions of placement events in Mandarin. Two general placement verbs, fang4 'put' and na2 'take', have large extensional categories, but their use is not unconstrained and Mandarin requires specific verbs to encode certain kinds of events. Six groups of specific verbs are identified according to certain shared distinctive semantic features, as summarized in Table 2.

Table 2 shows that crucial semantic distinctions are made based on the Goal of the placement (e.g. dressing verbs), the spatial relationship between a Figure and a Ground object (e.g. tight-fitting), Manner of placement, intention and control of the Agent, and Path of motion. All of these verbs occur as the $\mathrm{V}_{1}$ of compound, but some are also used by themselves, as in (3). The compound as whole, rather than any of the component verbs alone, expresses the motion reading. 


\section{Jidong Chen}

Table 2. Summary of the high-frequency specific placement verbs in the elicited descriptions of placement events

\begin{tabular}{|c|c|c|c|}
\hline Groups & Verbs & Glosses & Distinctive semantic features \\
\hline \multirow{3}{*}{$\begin{array}{l}\text { (un)dressing } \\
\text { verbs }\end{array}$} & tuol & take off & Figure: Clothing \\
\hline & chuan1 & put on & Figure: Clothing; Goal: body trunk and feet \\
\hline & dai4 & put on & $\begin{array}{l}\text { Figure: Clothes; Goal: extremities or joints } \\
\text { of body (e.g. hand, head, wrist) }\end{array}$ \\
\hline \multirow{6}{*}{$\begin{array}{l}\text { Verbs } \\
\text { specifying } \\
\text { relationship } \\
\text { between } \\
\text { Figure and } \\
\text { Ground }\end{array}$} & $b a 2$ & pull & $\begin{array}{l}\text { Relation between Figure and Goal (tight- } \\
\text { fitting); direction of motion (out) }\end{array}$ \\
\hline & choul & $\begin{array}{l}\text { pull, draw } \\
\text { (from long } \\
\text { container) } \\
\end{array}$ & $\begin{array}{l}\text { Relation between Figure and Goal (tight- } \\
\text { fitting); direction of motion (out) }\end{array}$ \\
\hline & chal & insert & $\begin{array}{l}\text { Relation between Figure and Goal (tight- } \\
\text { fitting); direction of motion (in); Figure } \\
\text { (rigid) }\end{array}$ \\
\hline & sail & stuff & $\begin{array}{l}\text { Relation between Figure and Goal (tight- } \\
\text { fitting); physical property of Figure (soft) }\end{array}$ \\
\hline & tiel & paste & Relation between Figure and Goal (clinging) \\
\hline & jian3 & pick & $\begin{array}{l}\text { Relation between Source and Goal: From a } \\
\text { lower location to a higher location }\end{array}$ \\
\hline \multirow{3}{*}{$\begin{array}{l}\text { Verbs } \\
\text { specifying } \\
\text { Manner }\end{array}$} & taol & draw, dig & Manner of placement \\
\hline & $s a 3$ & spread & Manner of placement \\
\hline & zhual & $\begin{array}{l}\text { grasp, hold } \\
\text { tightly }\end{array}$ & Manner of placement (grasping) \\
\hline \multirow{5}{*}{$\begin{array}{l}\text { Verbs } \\
\text { specifying } \\
\text { control and } \\
\text { intention }\end{array}$} & diao4 & fall & Lack of control and intention \\
\hline & sa3 & spill & Lack of control and intention \\
\hline & dao4 & pour & Intention and control \\
\hline & rengl & throw & Intention and control \\
\hline & tuil & push & Intention and control \\
\hline \multirow{6}{*}{$\begin{array}{l}\text { Verbs } \\
\text { encoding } \\
\text { Instrument }\end{array}$} & bao4 & hold in arm & $\begin{array}{l}\text { Instrument (arm or arm-like instrument in a } \\
\text { holding posture) }\end{array}$ \\
\hline & lingl & $\begin{array}{l}\text { carry in } \\
\text { hand }\end{array}$ & Instrument (hand or hand-like instrument) \\
\hline & $t i 2$ & $\begin{array}{l}\text { carry in } \\
\text { hand }\end{array}$ & Instrument (hand or hand-like instrument) \\
\hline & diaol & $\begin{array}{l}\text { hold in } \\
\text { mouth }\end{array}$ & Instrument (mouth) \\
\hline & xian2 & $\begin{array}{l}\text { hold in } \\
\text { mouth }\end{array}$ & Instrument (mouth) \\
\hline & jia2 & hold tightly & $\begin{array}{l}\text { Instrument (tight-fitting between Figure and } \\
\text { Instrument) }\end{array}$ \\
\hline \multirow{2}{*}{$\begin{array}{l}\text { Verb } \\
\text { encoding } \\
\text { Path }\end{array}$} & diao4 & fall & Path (downward) \\
\hline & shen 1 & $\begin{array}{l}\text { extend, } \\
\text { stretch }\end{array}$ & Path (forward) \\
\hline
\end{tabular}


Putting and Taking Events in Mandarin Chinese

\section{Conclusion}

This paper demonstrates the Mandarin-specific organization of the semantic components in the domain of placement events and the semantic extensional patterns of the general placement verbs and the specific verbs. It shows that Ground (Source/Goal) and Figure are encoded as arguments or adjunct coverb phrases and that Path and Motion are frequently encoded with directional verb compounds. A directional verb compound is composed of two or three free verbs in the form of $V_{1} V_{2}$ or $V_{1} V_{2} V_{3}$, with each verb encoding certain aspect of the motion event. The verb compounds as a whole convey the motion meaning of the placement. The verbs in the $\mathrm{V}_{1}$ slot, an open set of verbs, encompass general placement verbs fang4 'put' or na2 'take', a range of specific verb that make fine-grained semantic distinctions, and two verbs that entail the Path of motion. The verbs in the $V_{2}$ or $V_{3}$ slot form a closed set of two types of Path verbs: Trajectory verbs and deictic verbs.

This study contributes to the crosslinguistic study of typology of motion events. It supports the refined typology of motion events as proposed by Slobin (2004) that Mandarin, different from the satellite-framed languages (such as English and German), belongs to the "equipollently-framed languages". In this pattern, Path and Manner are expressed by equivalent grammatical forms (i.e. two or three free verbs in the compound).

\section{References}

Bennett, Paul. 1981. The Evolution of Passive and Disposal Sentences. Journal of Chinese Linguistics 9: 63-90.

Bowerman, Melissa, Gullberg, Marianne, Majid, Asifa, and Narasimhan, Bhuvana. 2004. The Crosslinguistic Encoding of Placement Events. In A. Majid, ed., Field Manual 2004, 10-24. Nijmegen, NL: Language \& Cognition Group, Max Planck Institute for Psycholinguistics.

Chao, Yuen-Ren. 1968. A Grammar of Spoken Chinese. Berkeley, CA: University of California.

Goldberg, Adele. 1995. Constructions: A Construction Grammar Approach to Argument Structure. Chicago: University of Chicago Press.

Haiman, John. 1985. Iconicity in Syntax. Amsterdam: John Benjamins.

Huang, Cheng-Teh James. 1982. Logical Relations in Chinese and the Theory of Grammar. Unpublished doctoral dissertation, MIT, Cambridge, MA.

Li, Charles, and Thompson, Sandra. 1981. Mandarin Chinese: A Functional Reference Grammar. Los Angeles, CA: University of California Press. 


\section{Jidong Chen}

Sun, Chao Fen, and Givón, Talmy. 1985. On the So-Called Sov Word Order in Chinese: A Quantified Text Study and Its Implications. Language 61: 329-351.

Tai, James Hao-Yi. 1985. Temporal Sequence and Chinese Word Order. In J. Haiman, ed., Iconity in Syntax, 49-72. Amsterdam: John Benjamins.

Talmy, Leonard. 1985. Lexicalization Patterns: Semantic Structure in Lexical Forms. In T. Shopen, ed., Language Typology and Syntactic Description, Vol. 3: Grammatical Categories and the Lexicon, 36-149. Cambridge, UK: Cambridge University Press.

Talmy, Leonard. 2000. Chapter 3: A Typology of Event Integration. Toward a Cognitive Semantics, Vol. 2: Typology and Process in Concept Structuring, 213-288. Cambridge, MA: MIT Press.

Tsao, Feng-Fu. 1996. On Verb Classification in Chinese. Journal of Chinese Linguistics 24: 138-191.

Wang, Li. 1954. Zhongguo Yufa Lilun (Theories of Chinese Syntax). Shanghai: Commercial Press.

Jidong Chen

California State University at Fresno

Department of Linguistics

5245 North Backer Avenue

M/S PB92, Fresno CA, 93740-8001

jchen@csufresno.edu 